\title{
Hábitos de estudio y rendimiento académico en Ingeniería de Sistemas
}

\author{
Study habits and academic performance in Systems Engineering
}

\author{
Eduar Bayona I. \\ Universidad Francisco de Paula Santander \\ Correo: ebayonai@ufpso.edu.co
}

Fecha de recibido: 08/02/2017 y Fecha de aprobación: 12/04/2017

\begin{abstract}
Resumen
Son diferentes los factores que influyen en el rendimiento académico de los estudiantes, en la presente investigación se evaluó qué tanto los estudiantes conocen su oficio para determinar si existe correlación entre los hábitos de estudio y el rendimiento académico. Se recopilaron investigaciones a nivel nacional e internacional, encontrando que son muy pocas las investigaciones sobre el tema de hábitos de estudio y el rendimiento académico, debido al error de considerar que los estudiantes cuando ingresan a la universidad ya cuentan con hábitos de estudio bien estructurados [1].

Para determinar el grado en el que los estudiantes conocen su oficio se aplicó la prueba de Pozar denominada Inventario de Hábitos de Estudio (IHE), se realizaron dos mediciones, la primera en el 2014 y la segunda en el 2016. Al momento de hacer el comparativo entre la aplicaciones de la prueba se encontró que los estudiantes del programa de Ingeniería de Sistemas de la Universidad Francisco de Paula Santander, Ocaña, cuentan con una condición buena en la categoría de condiciones ambientales de estudio, mostrando que se encuentran a gusto tanto en el ambiente familiar como el académico; aunque sigue siendo preocupante el tema de deserción, siendo del 45,85 por ciento en el momento de aplicar la segunda medición del IHE de Pozar en el año 2016, causada por el bajo rendimiento académico, lo que indica que se debe hacer mayor esfuerzo para disminuir las cifras.
\end{abstract}

Palabras Clave:

Hábitos de estudio Métodos de estudio Rendimiento académico Técnicas de estudio Inventario de hábitos y Pozar

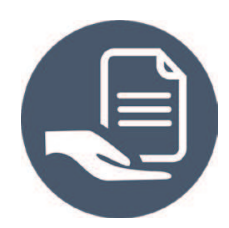

¥Se concede autorización para copiar gratuitamente parte o todo el material publicado en la Revista Colombiana de Computación siempre y cuando las copias no sean usadas para fines comerciales, y que se especifique que la copia se realiza con el conocimiento de la Revista Colombiana de Computación. 
Abstract

There are different factors influencing students' academic performance, in the present research it was assessed that both students know their trade and determine if there is correlation between study habits and academic performance. Research was collected at the national and international levels, finding that there is very little research on the subject of study habits and academic performance, as stated by due to the error of considering that students when they enter University students already have well-structured study habits [1].

In order to determine the degree to which students know their trade, the Pozar Study Habits Inventory (IHE) test was applied, two measurements were taken, one in 2014 and the other in 2016. In the test 2016, it was found that the students the of program of System ingenieering program of the University Francisco de Paula Santander, Ocaña section have a good condition in the category of environmental conditions of study, showing that they are at home in both the academic and the family environment; Although the issue of desertion continues to be worrying, being 45.85 percent at the time of applying the second IHE Pozar measurement in 2016, caused by poor academic performance, indicating that greater effort must be made to Decrease the figures.
Keywords:

Study Habits

Study Methods

Academic Performance

Study techniques

Habit inventory y Pozar.

\section{Introducción}

Los diferentes cambios que desde inicio de este milenio han acompañado a la educación superior: condiciones de calidad, registro calificado, procesos de certificación y acreditación, créditos académicos, flexibilidad curricular, entre otros, resaltan la importancia del autoaprendizaje, el trabajo independiente de los estudiantes en su proceso de formación y se modifican, no solo las dinámicas institucionales en el proceso educativo, sino tambien las dinámicas de aprendizaje de los estudiantes. Lo anterior hace que los sujetos aborden nuevos hábitos y procedimientos en la forma de estudiar; que trasciendan la costumbre de memorizar una serie de datos de un determinado texto o de una explicación o información impartida por el docente.

Estudiar como responsabilidad fundamental (pero no única) del estudiante en su proceso de formación, requiere de un método de estudio que le permita de forma lógica, gradual y sistemática enfrentar la información, conocimientos que le permitan aprender, comprendiendo lo que enfrenta en su estudio. No todos los estudiantes hacen frente con éxito a los nuevos desafíos que la Universidad plantea: aumento de la exigencia, necesidad creciente de organización del trabajo académico, desarrollo de procesos cognitivos y metacognitivos, mayor dedicación al estudio, autonomía [1]. La definición, apropiación y puesta en acción de un método de estudio por parte del estudiante en su proceso de formación, requiere de la definición de hábitos de estudio que le permitan abordar su propio método de estudio, enfrentar su desempeño académico y su aprendizaje. Los hábitos se empiezan a establecer desde los siete u ocho años y estos dependen de otros hábitos como la concentración, el orden y la atención, el sujeto que ha crecido respetando límites, rutinas y hábitos (sueño, alimentación e higiene) no tendrá dificultad para adquirir el hábito de estudiar.

El hábito de estudio es una acción que se realiza todos los días aproximadamente a la misma hora, la reiteración de esta conducta en el tiempo va generando un mecanismo inconsciente; los hábitos de estudio son un conjunto de rutinas de trabajo intelectual que afectan a las funciones de motivación, condiciones físicas y destrezas instrumentales básicas para el estudio; cada una de éstas proporciona elementos que permiten un adecuado desenvolvimiento del estudiante en el quehacer educativo, así como en su contexto personal [1]. Los hábitos de estudio como la práctica constante de las mismas actividades requieren de acciones cotidianas, las cuales serán con el tiempo un hábito afectivo siempre y cuando sean asumidas con responsabilidad, disciplina y orden. Se considera que el concepto de hábito de estudio está referido al modo como el individuo se enfrenta cotidianamente a su quehacer educativo, es la costumbre natural de procurar aprender permanentemente, lo cual implica la forma en la que el individuo se organiza en cuanto a tiempo, espacio, técnica y métodos concretos que utiliza para estudiar". Como se puede apreciar la repetición de la conducta dirigida al aprendizaje, enmarcada en unas condiciones que direccionen la conducta al éxito escolar, caracterizan la definición de hábito de estudio. 
Otra variable objeto de estudio en la presente investigación fue el rendimiento académico, el cual es definido como las calificaciones que obtuvo el estudiante por el aprendizaje adquirido en una asignatura, materia o curso [1]. El rendimiento académico es un indicador del éxito frente a las demandas de la formación docente, es una medida de las capacidades respondientes en forma estimativa, es decir lo que una persona ha aprendido como consecuencia de un proceso de formación [3]. Desde la perspectiva del estudiante, el rendimiento académico se define como la capacidad respondiente del alumno frente a estímulos educativos, la cual es susceptible de ser interpretada según propósitos establecidos. Atendiendo a lo anterior, se asumió en el estudio el rendimiento académico valorado desde el promedio ponderado del estudiante en el semestre académico.

\section{Metodología}

Se usa la recolección de datos para probar hipótesis, con base en la medición numérica y el análisis estadístico, para establecer patrones de comportamiento y probar teorías. Al final, con los estudios cuantitativos se pretende explicar y predecir los fenómenos investigados, buscando regularidades y relaciones causales entre elementos. Esto significa que la meta principal es la construcción y demostración de teorías (que explican y predicen). Del mismo modo, utiliza la recolección y el análisis de datos para contestar preguntas de investigación establecidas previamente y confía en la medición numérica, el conteo y frecuentemente en el uso de estadísticas para establecer con exactitud, patrones de comportamiento en una población.

Se estableció la aplicación del Instrumento Hábitos de Estudio de Pozar (2014) 10a edición revisada y ampliada por Tea ediciones Madrid. El diseño de la investigación es correlacional [4], su propósito, "es medir el grado de relación que existe entre dos o más variables en un contexto en particular"; se orienta a la perspectiva empírico-analítica, enfatiza el contexto de justificación o contrastación de hipótesis, siendo no experimental o ex postfacto ya que se trata de: "observar fenómenos tal y como se da en su contexto natural para después analizarlos" [5].

Para abordar el objetivo que refiere al tema de describir los métodos de estudio empleados por los estudiantes, se usó un diseño de investigación descriptivo de tipo longitudinal. El rendimiento académico se determinó mediante el promedio de calificaciones ponderado al concluir el semestre cursado, en el primer semestre y luego en el tercer semestre de la misma población de estudiantes objeto de nuestro estudio.

Para dar cumplimiento a los objetivos de la investigación, se aborda la Institución de Educación Superior, Universidad Francisco de Paula, del municipio de Ocaña, departamento Norte de Santander en la que se ofertan programas académicos en las áreas: Administrativas, Agrarias, Ingenierías, Educación y Artes. La población se encuentra conformada por los estudiantes del primer semestre del programa académico de Ingeniería de Sistemas en el año 2014 y en la primera medición participaron 50 estudiantes; en la segunda medición se aplicó nuevamente el instrumento a 21 estudiantes que previamente presentaron la medición 1, como se aprecia en la Tabla 1.

Estudiantes que conformaron la muestra:

\begin{tabular}{|c|c|c|}
\hline \multirow{2}{*}{ Carrera } & \multicolumn{2}{|c|}{ Número de Estudiantes } \\
\cline { 2 - 3 } & Medición 1 (2014) & Medición 2 (2015) \\
\hline Ingeniería de Sistemas & 50 & 21 \\
\hline
\end{tabular}

Tabla 1. Estudiantes del programa de Ingeniería de Sistemas. 


\section{Resultados}

En la medición 1, la aplicación de la herramienta de Pozar IHE permitió evidenciar que los estudiantes de Ingeniería de Sistemas cuentan con buenas condiciones ambientales (I) para el estudio, pero con tendencia normal en lo que se refiere a las características planificación del estudio (II), utilización de materiales (III), asimilación de contenidos (IV) y un grado de sinceridad (V) normal. El promedio del rendimiento académico para la medición 1 se estableció en 3,2.

\begin{tabular}{|c|c|c|c|c|c|c|c|c|c|c|}
\hline \multicolumn{11}{|c|}{ PERFIL } \\
\hline \multirow{2}{*}{ Escala } & \multirow{2}{*}{ P.D } & \multirow{2}{*}{$\frac{\mathrm{Mal}}{1}$} & \multicolumn{2}{|c|}{ No Satisfactorio } & \multicolumn{3}{|c|}{ Normal } & \multicolumn{2}{|c|}{ Bien } & \multirow{2}{*}{$\begin{array}{c}\text { Excelente } \\
9\end{array}$} \\
\hline & & & 2 & 3 & 4 & 5 & 6 & 7 & 8 & \\
\hline I & 7 & 0 & O & O & 0 & (1) & 2 & - & 0 & 0 \\
\hline II & 5 & 0 & 0 & 0 & 0 & 8 & 0 & $\mathrm{C}$ & 0 & 0 \\
\hline III & 5 & 0 & 0 & O & 0 & $D$ & 0 & C & 0 & 0 \\
\hline IV & 5 & 0 & ( ) & 0 & 0 & 2 & 0 & C & 0 & 0 \\
\hline V & 4 & 0 & 0 & 0 & 3 & 0 & 0 & C & 0 & 0 \\
\hline
\end{tabular}

Figura 1. Perfil de los estudiantes del programa de Ingeniería de Sistemas, medición 1.

\begin{tabular}{|c|c|c|c|c|c|c|c|c|c|c|}
\hline \multicolumn{11}{|c|}{ PERFIL } \\
\hline \multirow{2}{*}{ Escala } & \multirow{2}{*}{ P.D } & \multirow{2}{*}{$\frac{\mathrm{Mal}}{1}$} & \multicolumn{2}{|c|}{ No Satisfactorio } & \multicolumn{3}{|c|}{ Normal } & \multicolumn{2}{|c|}{ Bien } & \multirow{2}{*}{$\begin{array}{c}\text { Excelente } \\
9\end{array}$} \\
\hline & & & 2 & 3 & 4 & 5 & 6 & 7 & 8 & \\
\hline I & 6 & 0 & O & O & 0 & & C) & 0 & O & 0 \\
\hline II & 5 & 0 & ( & O & 0 & 7 & 0 & 0 & 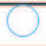 & 0 \\
\hline III & 5 & 0 & $\mathrm{O}$ & O & O & 7 & O & 0 & O & O \\
\hline IV & 5 & 0 & O & 0 & O & & O & ( & O & O \\
\hline V & 5 & 0 & (1) & O & $\mathrm{O}$ & 1 & O & O & O & ( \\
\hline
\end{tabular}

Figura 2. perfil de los estudiantes del programa de Ingeniería de Sistemas, medición 2.

En la medición 2 se aprecia poca diferencia en el cambio de hábitos de los estudiantes, se disminuye el nivel de condiciones ambientales (I), el grado de sinceridad mejora de nivel pasando del 4 al 5 aunque se mantiene en la categoria normal, el rendimiento académico disminuyó pasando de 3,3 a 3,2.

\subsection{Correlación entre hábitos de estudio y rendimiento académico}

\subsubsection{Hipótesis}

$\mathrm{H}^{\circ}=$ el rendimiento académico es independiente de los hábitos de estudio

$\mathrm{Ha}=$ el rendimiento académico depende de los hábitos de estudio

\section{Condiciones:}

Si R es menor que 0 la hipótesis $\mathrm{H}^{\circ}$ es verdadera, de lo contrario $\mathrm{Ha}$ es verdadera

Determinando la correlación:

Tomando como referencia el tamaño de los datos fruto del análisis, (21) y con el objetivo de buscar relaciones entre las variables de hábitos de estudio y rendimiento Académico 


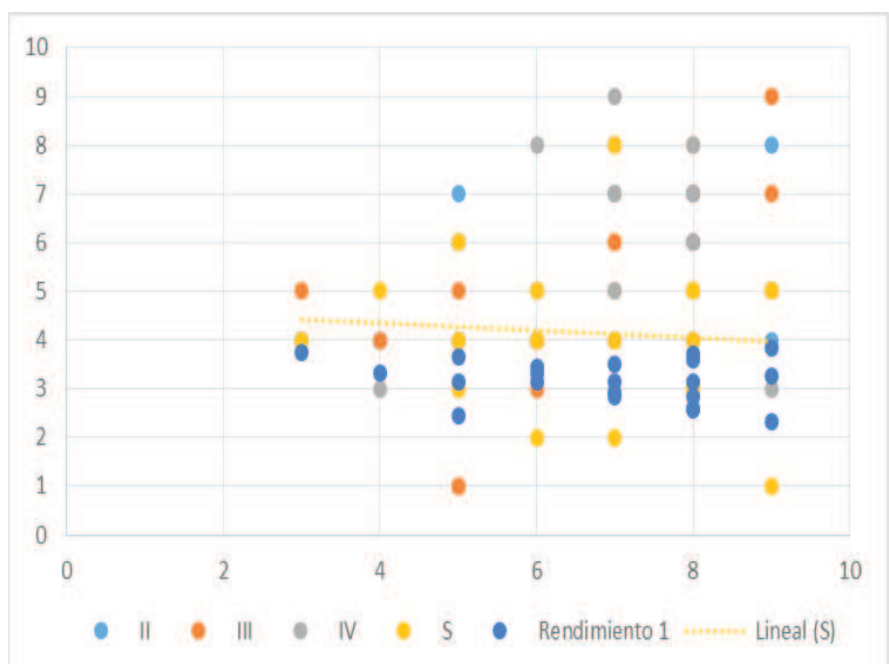

Figura 3. Dispersión entre rendimiento académico y hábitos de estudio, medición 1.

En la Figura 3., se aprecia que no existe correlación entre el rendimiento académico y los hábitos de estudio, datos que corresponden a la medición 1.

\begin{tabular}{crrrrrc}
\hline & I & \multicolumn{1}{c}{ II } & III & IV & S & Rendimiento 1 \\
\hline I & 1,00 & & & & & \\
II & 0,36 & 1,00 & & & & \\
III & 0,56 & 0,35 & 1,00 & & & \\
IV & 0,16 & 0,09 & 0,24 & 1,00 & & \\
S & $-0,09$ & $-0,33$ & $-0,19$ & $-0,35$ & 1,00 & \\
Rendimiento 1 & $-0,22$ & $-0,08$ & 0,33 & 0,10 & 0,05 & 1,00 \\
\hline
\end{tabular}

Tabla 2. Coeficiente de correlación entre rendimiento académico y hábitos de estudio medición 1. Fuente: Elaboración propia.

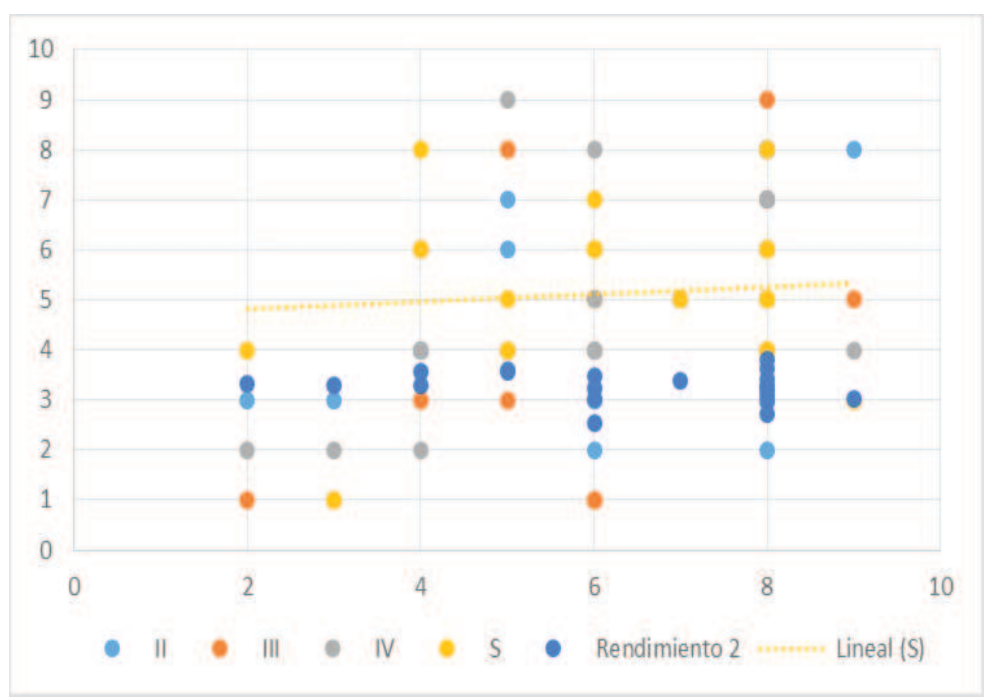

Figura 4. Dispersión rendimiento académico y hábitos de estudio, medición 2.

En la Figura 4, al igual que la Figura 3 se evidencia que no existe correlación entre las variables de rendimiento académico y los hábitos de estudio. 


\begin{tabular}{lcccccc}
\hline & I & II & III & IV & S & Rendimiento 2 \\
\hline II & 1,00 & & & & & \\
II & 0,57 & 1,00 & & & & \\
III & 0,55 & 0,66 & 1,00 & & & \\
IV & 0,59 & 0,56 & 0,41 & 1,00 & & \\
S & 0,09 & $-0,25$ & $-0,07$ & 0,14 & 1,00 & \\
Rendimiento 2 & $-0,21$ & $-0,09$ & $-0,10$ & 0,01 & 0,04 & 1,00 \\
\hline
\end{tabular}

Tabla 3. Coeficiente de correlación entre rendimiento académico y hábitos de estudio medición 2.

El coeficiente de correlación es $\mathrm{R}=0,0253$ valor que tiende a 0 ratificando la hipótesis $H^{\circ}$ que se refieren a que el rendimiento académico es independiente de los hábitos de estudio

\section{Discusión}

La carencia de investigaciones relacionadas con los hábitos de estudio en la educación superior muestra la importancia de hacer investigaciones que se orienten a determinar las causas que afectan al rendimiento académico, que le permitan a las Instituciones tomar decisiones oportunas que se encaminen a la calidad efectiva [1].

Luego de realizar diferentes estudios para conocer la existencia de las investigaciones que se relaciones con el rendimiento académico y los hábitos de estudio, se pueden nombrar las investigaciones de [3], [5], [10] [11] [12] [13] donde se ha determinado que no son suficientes, debido a la multitud de cambios y oportunidades que se han generado, en su mayor parte gracias a la tecnología. Debido a lo complejo del problema el instrumento de Pozar contribuye a dar "un primer paso de aproximación al problema", ya que tiene como objetivo "abrir brecha, definiendo las actitudes del estudiante hacia su trabajo. La tarea esencial, la importante y verdaderamente orientadora, viene después cuando el educador interviene sobre estos aspectos".

El nuevo interrogante que se genera en cuando a las investigaciones Pozar, tiene que ver con la funcionalidad de los baremos, aunque el autor argumenta que no existían diferencias suficientemente relevantes que justificaran una nueva baremación y que el uso de los baremos ya existentes permitía una buena evaluación de los hábitos de estudio en los escolares. Es claro que algo se tiene que hacer, pues en la presente investigación se determinó que no existe relación entre el rendimiento académico y los hábitos de estudio .

El aporte de la investigación permite hacer un acercamiento, conocer cómo es que los estudiantes desempeñan su responsabilidad académica, las técnicas y métodos que más usan y la efectividad o no en sus resultados [14].

Mediante la aplicación de la prueba de Pozar IHE a estudiantes del programa de Ingeniería de Sistemas de la Facultad de Ingenierías, de la Universidad Francisco de Paula Santander, Ocaña, en las dos mediciones; la primera realizada cuando inician su vida universitaria y la segunda cuando ha trascurrido un año, el perfil muestra que el grupo en general presenta un resultado normal, es decir, muy poco o casi nada en la aplicación de hábitos de estudio por parte de los estudiantes, encontrando que existen aspectos que se deben mejorar en cada una de las escalas. Para el caso de las condiciones ambientales la calificación es buena bajo, indicando que el grupo en cuanto a las condiciones físicas, personales, comportamiento académico y rendimiento, cumple satisfactoriamente; por lo tanto se debe mantener o mejorar, se recomienda leer y aplicar el instrumento "Aprender a Estudiar" [6] .

En la Planificación del Estudio, la clasificación es normal; indicando que tanto en la Organización como en Horarios se cumple satisfactoriamente; para mejorar los resultados se recomienda leer y aplicar la herramienta "Aprender a Estudiar" [7] [16] . En el empleo de los materiales se obtuvo una calificación normal en lectura, libros y resúmenes, se recomienda una lectura a la herramienta "Didáctica del Estudio". En la escala de asimilación de contenidos se cumple satisfactoriamente, pero es necesario mantener los resultados o mejorarlos, se recomienda leer la "Didáctica del Estudio", que servirá para el perfeccionamiento de los métodos aplicados. Para la asimilación de contenidos la 
calificación es Normal para los aspectos de memorización, trabajo individual y en equipo, se debe mantener o mejorar mediante la aplicación de las normas "Aprender a Estudiar" [7], del mismo modo es necesario tener presente el papel protagónico que juega la voluntad o interés de los estudiantes, docentes y padres de familia para lograr buenos resultados en el rendimiento académico, Covey[17].

La calificación normal de la categoría de Sinceridad, garantiza que las respuestas de cada uno de los estudiantes han sido bastante sinceras. "Con este diagnóstico se puede emprender una "campaña" de mejora de los hábitos de trabajo y estudio proporcionando a los alumnos las normas concretas de actuación" [7].

Los estudiantes cuentan con conocimiento y aplicación de las técnicas de estudio, pero no lo hacen con la rigurosidad necesaria, en algunos casos lo aplican previamente para presentar un examen, sin que se genere hábito, siendo el bajo rendimiento académico una consecuencia del desconocimiento de un adecuado manejo de métodos y técnicas de estudio en concordancia con lo manifestado por [1].

Los resultados descritos en este estudio llevan a establecer unos cuestionamientos en los cuales se debe resaltar la relación de los nuevos estilos de aprendizajes a las nuevas tendencias de estudio, utilizadas por los estudiantes, los cuales difieren de los hábitos de estudio, tradicionalmente reconocidos, como los relacionados a través del cual se orienta el inventario de hábitos de estudio, el cual tiene como finalidad la evaluación de los hábitos de estudio que influyen en las tareas de aprendizaje en cuanto a cuatro aspectos fundamentales: las condiciones ambientales del estudio, la planificación del estudio, la utilización de materiales y la asimilación de contenidos.; ya que en la actualidad los estudiantes no necesariamente deben estudiar en ambientes controlados, muy bien iluminados, sin ruidos, sin que esto les pueda asegurar ser lo más conveniente para realizar dichos estudios en la actualidad, ya que se debe tener en cuenta que los estudiantes cuentan en su gran mayoría con índices de atención dispersos y esto les permite desarrollar sus estudios en ambientes con diferentes estímulos en especial tecnológicos como lo son la música, el WhatsApp, el internet entre otros.

\section{Conclusiones}

En la presente investigación los hábitos de estudió no se correlacionaron con el rendimiento académico, lo que significa que para tener buen rendimiento no necesariamente se deben tener buenos hábitos de estudio, ratificando lo afirmado por las investigaciones de Villegas y Muñoz; Picasso, Villanelo y Lorenzo y De la Peña, situación que es contraria a lo manifestado por los autores: Pozar, Martínez y Torres, Ríos y Ramos quienes concluyeron en su momento que el bajo rendimiento se debe en general al desconocimiento de un adecuado manejo de métodos y técnicas de estudio, a su vez consideraron que los hábitos de estudio inciden positivamente en el rendimiento académico y llegaron a establecer la correlación entre las dos variables.

Las cosas hoy en día parecen cambiar ya que desde hace unos pocos años la tecnología ha entrado a hacer parte del diario vivir, los jóvenes ya disponen de dispositivos conectados a redes sociales como Facebook o WhatsApp, que sin duda están causando cambios en los hábitos de los estudiantes; más aún pueden afectar de manera positiva o negativa el estudio, dependiendo del buen o mal uso que se le dé al dispositivo; conviene desarrollar y orientar las habilidades tecnologicas con las que cuentan los estudiantes para lograr el aprendizaje significativo en el aula.

El resultado de aplicar el inventario de hábitos de estudio evidenció que los estudiantes del Programa académico de Ingeniería de Sistemas presentan poco o casi ningún hábito de estudio [7], los estudiantes deben corregir sus hábitos para lograr terminar su formación profesional con éxito, de igual forma se debe considerar lo manifestado por Nuñez y Sanchez, quienes determinaron que existen otros factores que intervienen en el estudio: Aspectos como la personalidad, motivación y aptitudes e intereses, que deben considerase ya que pueden llegar a afectar significativamente el estudio. Del mismo modo, se debe tener en cuenta que los tiempos han cambiado, hoy en día los contextos se acceleran cada vez más, donde la tecnología juega un papel protagonico, de ahí la importancia de considerar cómo interviene en el 
aprendizaje para aprovecharla. Los resultados obtenidos en la investigación muestran que existe una gran oportunidad en cuanto a propuestas pedagógicas encaminadas a desarrollar los hábitos de estudio para mejorar el rendimiento académico del programa de Ingeniería de Sistemas en la Universidad Francisco de Paula Santander, Ocaña.

El éxito del estudio depende en gran medida de la volutad o fuerzas que se fijan desde los actores: estudiantes, profesores y familia, como lo afirma Covery [17] .

\section{Referencias}

[1] U. d. I. Andes, «Determinantes de la Deserción,» Bogotá , 2014.

[2] C. Núnez y J. C. Sánchez, "Hábitos de estudio y rendimiento en EGB y BUP. Un estudio comparativo,» Complutense de Educación, p. 43, 1991.

[3] F. M. d. B. y. J. H. D. Francisco Camarero Suárez, "Estilos y estrategias de aprendizaje en estudiantes universitarios,» Psicothema, pp. 615-622, 2000.

[4] Muñoz-Repiso, F. Tejedor y A. García-Valcárcel, «Causas del bajo rendimiento del estudiante universitario,» de Educación, pp. 443-473, 2007.

[5] F. Hernández, Metodología del Estudio, Bogotá: McGraw Hill, 2001.

[6] F. F. Pozar, Inventario de Hábitos de Estudio ( 10 Edición, revisada y ampliada), Madrid : T.E.A Ediciones, S.A.U, 2014.

[7] M. Torres, I. Tolosa, C. Urrea y A. Monsalve, Inventario de hábitos de estudio en una clase para toma, Bogotá: Cienc. Salud, 2009.

[8] H. P. Hernández y G. L. García, «Enfoques, métodos y procedimeintos en la psicología del estudio».

[9] A. Q. U. Fabiola Cruz Núñez, "Hábitos de estudio y rendimiento académico en,» actualidades investigativas en educación, p. 3, 2011.

[10] F. F. Pozar, Inventario Hábitos de Estudio, Madrid: TEA Ediciones, 2014.

[11] H. Sampieri, C. Fernández y P. Baptista, Metodología de la Investigación, México D.F: McGraw-Hili, 2006.

[12] J. Valenzuela y M. Flores, Fundamentos de investigación educativa, Volumen 2, México , 2012.

[13] E. L. Gross, Cómo aprender a Estudiar, Madrid : Libsa, 2014.

[14] F. Hernández, Metódos y Técnicas de Estudio en la Universidad, Bogotá: Presencia Ltda, 1988.

[15] L. Ebee, Cómo Aprender a Estudiar, Alcobendas: Libsa, 2014.

[16] F. Hernández, Metodología del Estudio, Bogotá: McGRAW- Hill, 2001.

[17] S. R. Covey, Los 7 hábitos de la Gente Altamente, Buenos Aires: Paidos, 1997.

[18] C. Hernández, N. Rodríguez y V. Ángel, «Los hábitos de Estudio y Motivación pra el Aprendizaje de los Alumnos en Tres Carreras de Ingeniería,» Revista de la Educación Superior , p. 71, 2012. 


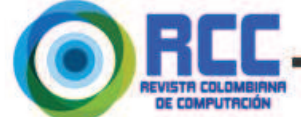

\section{Sobre los Autores}

Eduar Bayona Ibáñez. Especialista en Auditoría de Sistemas, Msc. Prácticas Pedagógicas. Docente ocasional Universidad Francisco de Paula Santander, Ocaña.

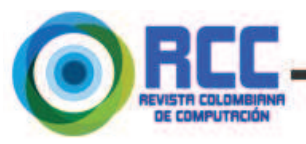

\section{Este artículo se cita:}

IEEE

B. I. Eduar, "Hábitos de estudio y rendimiento académico en Ingeniería de sistemas," Rev. Colomb. Comput., vol. 18, no. 1, pp. 6169, 2017.

APA

Eduar, B. I. (2017). Hábitos de estudio y rendimiento académico en Ingeniería de sistemas. Revista Colombiana de Computación, 18(1), 61-69. 